\title{
Influence of Off-resonance in myocardial T1-mapping using SSFP based MOLLI method
}

\author{
Peter Kellman ${ }^{1 *}$, Daniel A Herzka ${ }^{2}$, Andrew E Arai ${ }^{1}$ and Michael Schacht Hansen ${ }^{1}$
}

\begin{abstract}
Background: Myocardial T1-mapping methods such as MOLLI use SSFP readout and are prone to frequency-dependent error in T1-measurement. A significant error in T1 may result at relatively small off-resonance frequencies that are well within the region without banding artifacts.

Methods: The sensitivity of T1-estimates based on the SSFP based MOLLI sequence to errors in center frequency are calculated by means of a Bloch simulation and validated by phantom measurements. Typical off-resonance errors following local cardiac shimming are determined by field mapping at both 1.5 and 3.0T. In vivo examples demonstrate the artifactual appearance of T1-maps in the presence of off-resonance variation.

Results: Off-resonance varied $61.8 \pm 15.5 \mathrm{~Hz}$ (mean $\pm \mathrm{SD}, \mathrm{n}=18$ ) across the heart at $1.5 \mathrm{~T}$ and $125.0 \pm 40.6 \mathrm{~Hz}$ (mean $\pm \mathrm{SD}, \mathrm{n}=18$ ) at 3.0T. For $\mathrm{T} 1=1000 \mathrm{~ms}$, the variation in $\mathrm{T} 1$ due to off-resonance variation was approximately $20 \mathrm{~ms}$ at $62 \mathrm{~Hz}$, and $>50 \mathrm{~ms}$ at $125 \mathrm{~Hz}$.

Conclusions: Regional variations due to the inability to completely shim the B0-field variation around the heart appear as regional variation in $\mathrm{T} 1$, which is artifactual.
\end{abstract}

Keywords: T1 map, Error, Off-resonance, MOLLI, Shim, SSFP

\section{Background}

The frequency response of steady state free precession (SSFP) sequences is well known and results in dark band artifacts [1-3]. Myocardial T1-mapping methods such as the modified Look-Locker inversion recovery method (MOLLI) $[4,5]$ use SSFP readout and are therefore prone to frequency dependent errors in T1-measurements. The off-resonance behavior of SSFP and associated banding artifacts are often analyzed assuming the magnetization is at steady state as in continuous cine imaging. However, the MOLLI imaging sequence uses single shot imaging with data acquired on the approach to steady state. As a result, the off-resonance response becomes dependent on the initial condition, which is in turn dependent on the inversion recovery time. This leads to a frequency dependent change in the apparent inversion recovery. It is not well appreciated that a significant error in T1 may result at relatively small off-resonance frequencies that are well within the region without

\footnotetext{
* Correspondence: kellman@nih.gov

${ }^{1}$ National Heart, Lung, and Blood Institute, National Institutes of Health, DHHS, 10 Center Drive MSC-1061, Bethesda, MD 20892, USA

Full list of author information is available at the end of the article
}

banding artifacts. As T1-mapping and extra-cellular volume (ECV) mapping based on T1 measurement are used to detect more subtle cardiomyopathies [6-14], small errors in $\mathrm{T} 1$ become more significant. Hence, even small variations in $\mathrm{T} 1$ introduced by off-resonance due to the inability to completely shim B0-field variation may lead to significant biases in measured T1 that may be falsely confused with real pathology.

The readout excitation flip angle used in MOLLI is typically low $\left(35^{\circ}\right)$ compared to SSFP imaging as used for cine function imaging to reduce the influence of the readout on the inversion recovery. We hypothesize that reducing the flip angle improves T1-measurement accuracy with significant effect on the sensitivity to offresonance. In this work, the sensitivity of T1 measurement to off-resonance frequency and readout excitation flip angle are quantified for specific MOLLI protocols.

\section{Methods \\ Simulation}

A waveform level Bloch simulation of the MOLLI T1mapping method was implemented to study errors in

\section{Biomed Central}


T1-mapping, their dependencies and their sensitivities to various sequence and protocol design parameters. The simulation included the RF excitation pulse waveform and gradients in order to accurately model the variation in flip angle across the slice profile. Simulation results are provided for a specific MOLLI protocol which used a $5(3 \mathrm{~s}) 3$ sampling scheme (similar to protocol used in $[10,11])$ which uses 2 inversions with images acquired for 5 heartbeats following the first inversion, followed by a 3 second recovery period, and images acquired for 3 heartbeats following the second inversion. T1 fits are performed to the simulated magnetization using a 3parameter model and a Look-Locker correction is applied to the apparent $\mathrm{T} 1$ to correct for the influence of readout $[4,5,15]$. The T1-error due to the approximation used in the Look-Locker correction is the predominant error in this analysis [16]. Influence of magnetization transfer (MT) or imperfect inversion [17] are not considered here. Imaging parameters were: $256 \times 144$ matrix, $\mathrm{FA}=35^{\circ}$, TR $=$ $2.8 \mathrm{~ms}$, TImin $=105 \mathrm{~ms}$, TIshift $=80 \mathrm{~ms}$. A partial Fourier acquisition in the phase encode dimension was used to reduce the minimum achievable inversion time and reduced the overall shot duration. A partial Fourier factor of $7 / 8$ was used in the phase encode direction with 126 actual acquired lines (144 matrix size) plus 12 additional central lines for parallel imaging auto-calibration. The center of kspace was at 33 lines and there were 5 additional linear ramp flip angle pulses to reduce transient oscillations [18]. The T1 error was calculated for a range of T1's from $200-1200 \mathrm{~ms}$ at a fixed $\mathrm{T} 2=45 \mathrm{~ms}$. The errors were also simulated for the specific values of phantom $\mathrm{T} 1$ and T2 used for comparison with measurements.

Additional simulations were performed to assess the sensitivity of off-resonance errors to imaging protocol parameters. Sensitivity to off-resonance arises due to the influence of the SSFP readout during the approach to steady state. Both the total number of RF pulses (Ntotal) and the number of pulses to the center of k-space (Ncenter) will affect the T1-mapping accuracy and sensitivity to off-resonance. The measured phantom and invivo protocols used Ncenter/Ntotal: 33/75, based on a matrix size with 144 phase encodes, Fourier factor $=7 / 8$, and 12 extra central lines acquired for in-place autocalibration of parallel imaging (factor 2). A range of protocols were tested and results are provided for a significantly shortened Ncenter/Ntotal: $16 / 48$, based on a matrix size with 128 phase encodes, Fourier factor $=3 / 4$, and no extra central lines acquired corresponding to a separate reference line approach for auto-calibration.

\section{Phantom measurements}

Experimental validation was performed for a $\mathrm{CuSO} 4$ doped agar gel phantom using the specific MOLLI protocol at varying off-resonance frequencies in $10 \mathrm{~Hz}$ increments. Measured and simulated T1 are compared for a test tube phantom with $\mathrm{T} 1=1197 \mathrm{~ms}$ and $\mathrm{T} 2=47$ ms (1.5T Magnetom AERA, Siemens Medical Solutions, Erlangen, Germany).

\section{Field map measurements}

In-vivo measurements of off-resonance maps due to variation in $\mathrm{B} 0$-field were acquired to determine the typical expected variation in frequency. Measurements were made in $\mathrm{n}=18$ subjects referred for CMR assessment of known or suspected heart disease at both 1.5 (age $46 \pm 20$ ) and 3.0T (age $36 \pm 17$ ) using a multi-echo GRE sequence. Field maps were estimated as a byproduct of water fat separated image reconstruction [19]. A second order shim was performed over a local volume (box) encompassing the whole heart. Off-resonance frequency variation was measured in the left ventricle for a mid-ventricular, single short axis slice per subject.

\section{In-vivo imaging}

In-vivo T1-maps were acquired for normal healthy volunteers to illustrate the apparent variation in measured T1 with off-resonance. Images were acquired at several center frequencies to demonstrate the sensitivity. Imaging was performed on 1.5T Magnetom AVANTO and AERA scanners and 3T Magnetom SKYRA scanner (Siemens Medical Solutions, Erlangen, Germany). This study was approved by the local Institutional Review Board of the National Heart, Lung, and Blood Institute and all subjects gave written informed consent to participate.

\section{Results}

\section{Off-resonance variation}

Off-resonance frequency in the LV was measured at 1.5 and 3.0T after shimming over a local heart volume. At $1.5 \mathrm{~T}$, the mean off-resonance frequency in the LV myocardium was $20.3 \pm 13.0 \mathrm{~Hz}$. The maximum off-resonance in the LV was $61.8 \pm 15.5 \mathrm{~Hz}(\mathrm{n}=18)$. At 3.0T, the mean off-resonance frequency in the LV myocardium was $15.4 \pm 29.3 \mathrm{~Hz}$. The maximum offresonance in the $\mathrm{LV}$ was $125.0 \pm 40.6 \mathrm{~Hz}(\mathrm{n}=18)$.

\section{Simulation}

Figure 1 shows the response of SSFP readout vs frequency for various flip readout excitation angles shows the familiar dark bands spaced at $1 / \mathrm{TR}(\mathrm{T} 1 / \mathrm{T} 2 / \mathrm{TR}=1000 /$ $45 / 2.8 \mathrm{~ms}$ ). The left panel of Figure 1 shows the steady state response and the right panel shows the response for the transient approach to steady state corresponding to single shot imaging with initial full magnetization. The transient response has greater magnetization and different shape off-resonance response curves than the steady state response. The transient approach to steady state for single shot imaging during inversion recovery with different 

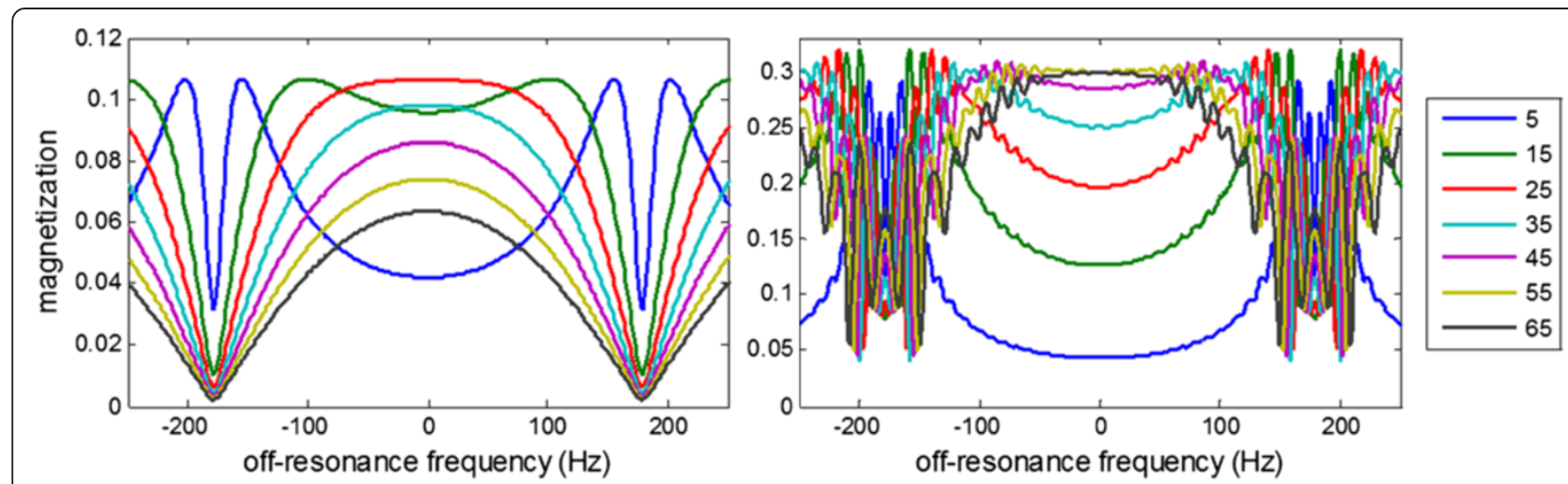

Figure 1 Off-resonance response for a variety of flip angles for SSFP at steady state (left) and during the approach to steady state (right) for $\mathbf{n}=\mathbf{3 3} \mathrm{RF}$ pulses to $\mathrm{k}$-space center with $\mathrm{TR}=\mathbf{2 . 8} \mathrm{ms}$ and $\mathbf{5}$ pulse linear run up. Note that the vertical axes have different scale since the steady state magnetization is reduced.

initial magnetization for each inversion time image has an off-resonance response (Figure 2, left) which distorts the apparent T1-recovery (Figure 2 right).

The off-resonance error in T1 is shown in Figure 3 for a MOLLI 5(3)3 protocol with 35 degree flip angle for a range of tissue T1 values with T2 $=45 \mathrm{~ms}$. Error in $\mathrm{ms}$ is displayed on the top panel of Figure 3 and in percent on the bottom panel. The T1 error and sensitivity to offresonance frequency increase for higher values of T1. At $\mathrm{T} 1=1000 \mathrm{~ms}$, measured $\mathrm{T} 1$ varied by $10 \mathrm{~ms}(1 \%)$ across \pm $50 \mathrm{~Hz}$, and $20 \mathrm{~ms}(2.0 \%)$ across $\pm 75 \mathrm{~Hz}$. This variation across frequency is in addition to the measurement error for on resonance tissue.

Sensitivity to off-resonance for a nominal T1 $=1000 \mathrm{~ms}$ was simulated for a modified protocol acquired with fewer RF pulses corresponding to smaller matrix size, higher partial Fourier factor, and eliminating in-place auto-calibration. The off-resonance sensitivity at $100 \mathrm{~Hz}$, i.e., difference between on- and off-resonant T1-estimates, was calculated to be $42 \mathrm{~ms}$ at $1000 \mathrm{~ms}$ (4.2\%) using the protocol with Ncenter/Ntotal $=33 / 75$, and reduced to $27 \mathrm{~ms}(2.7 \%)$ using the protocol with Ncenter/Ntotal = $16 / 48$. The off-resonance sensitivity at $50 \mathrm{~Hz}$ varied from $0.8 \%$ to $0.5 \%$, for the cases $33 / 75$ and $16 / 48$ respectively.

\section{Phantom measurements}

Experimental data is in close agreement with the simulation (Figure 4) in the calculation of off-resonance dependence of T1-estimation using MOLLI with SSFP readout.

\section{In-vivo examples}

An example study at $3 \mathrm{~T}$ demonstrates the sensitivity to off-resonance. In this example, a local $2^{\text {nd }}$ order shim is used (Figure 5) to minimize off-resonance variation. Despite shimming, there is a residual uncompensated off-resonance variation at regions near the tissue-air interface that creates a detectable change in apparent $\mathrm{T} 1$

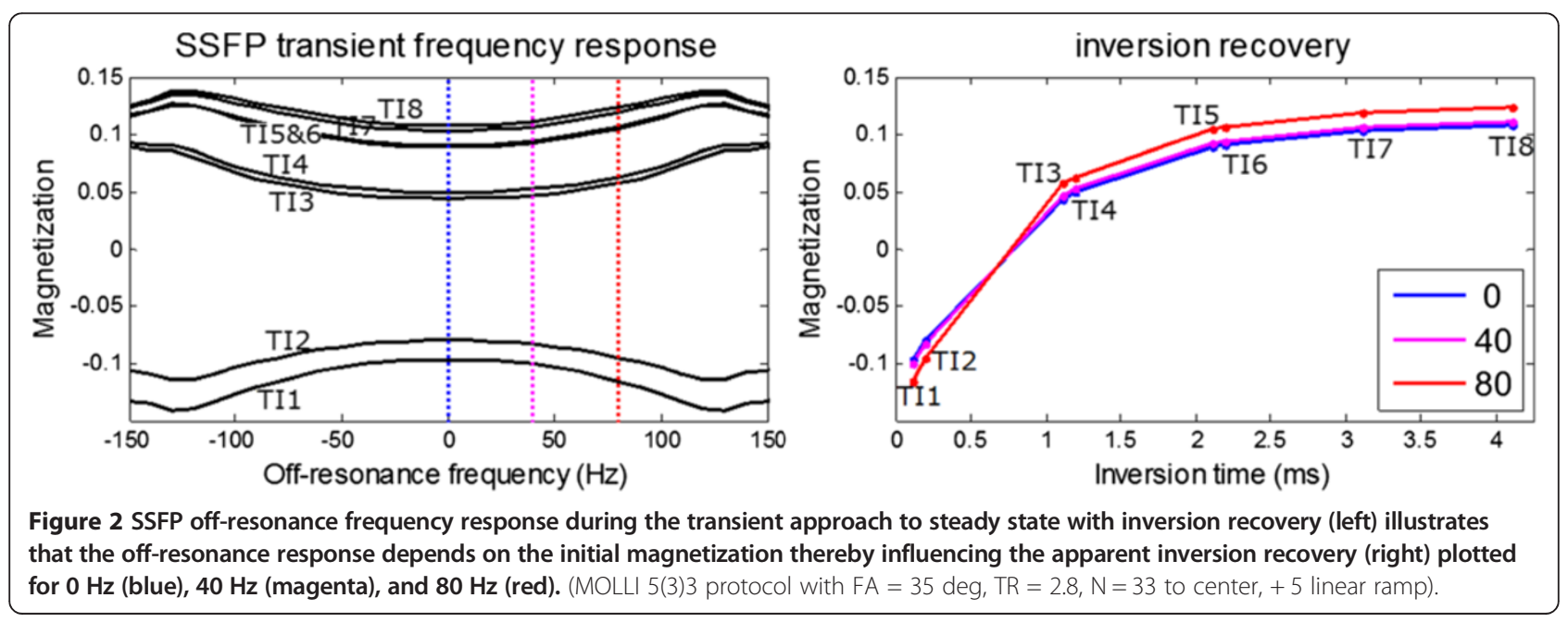



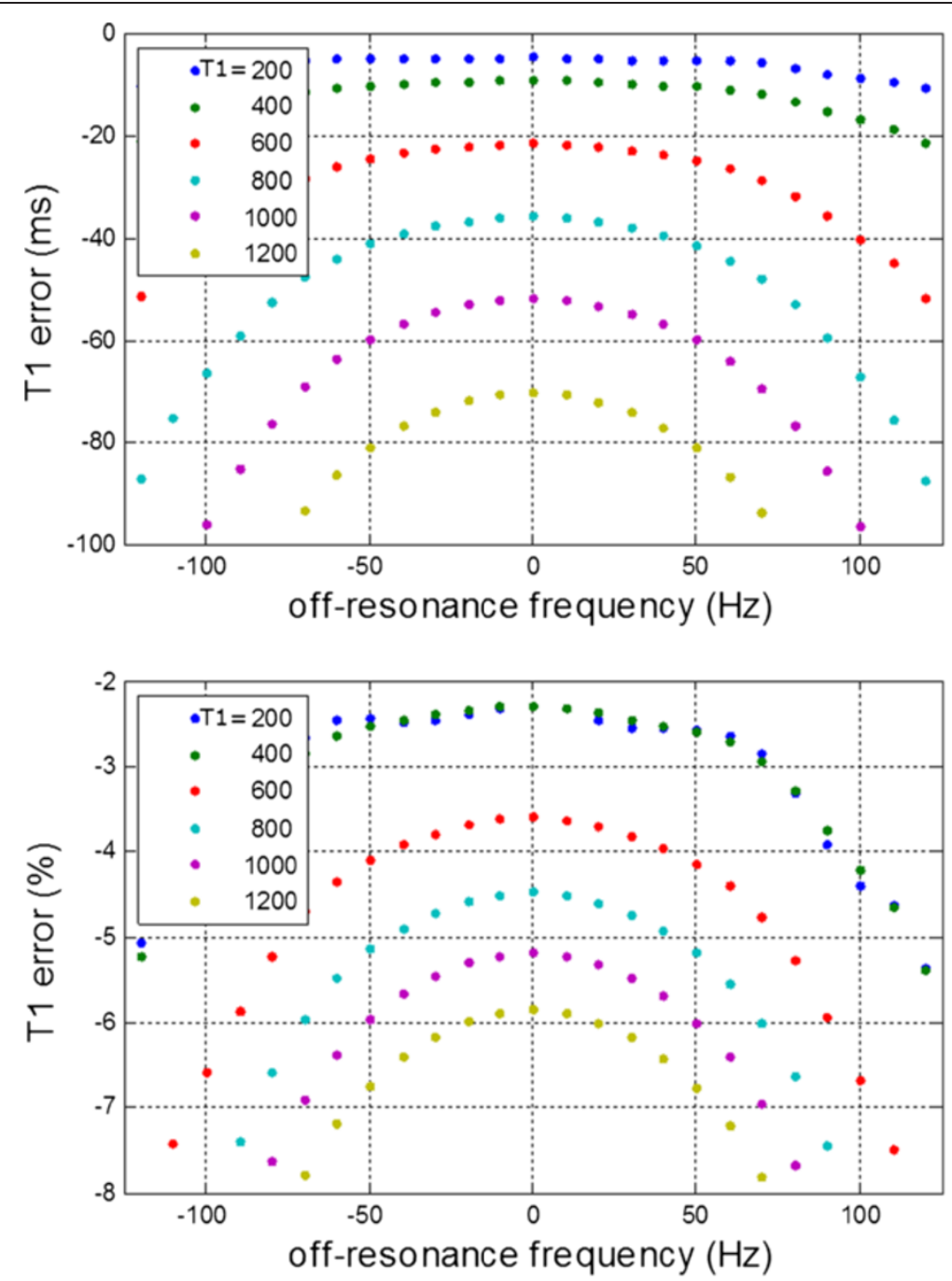

Figure 3 Off-resonance error in T1 using MOLLI 5(3)3 protocol with 35 degree flip angle for a range of tissue T1 values with T2= 45 ms, showing error in $\mathbf{m s}$ (top) and percent (bottom). The T1 error and sensitivity to off-resonance frequency increase for higher values of T1.

\section{T1 estimate vs off-resonance}

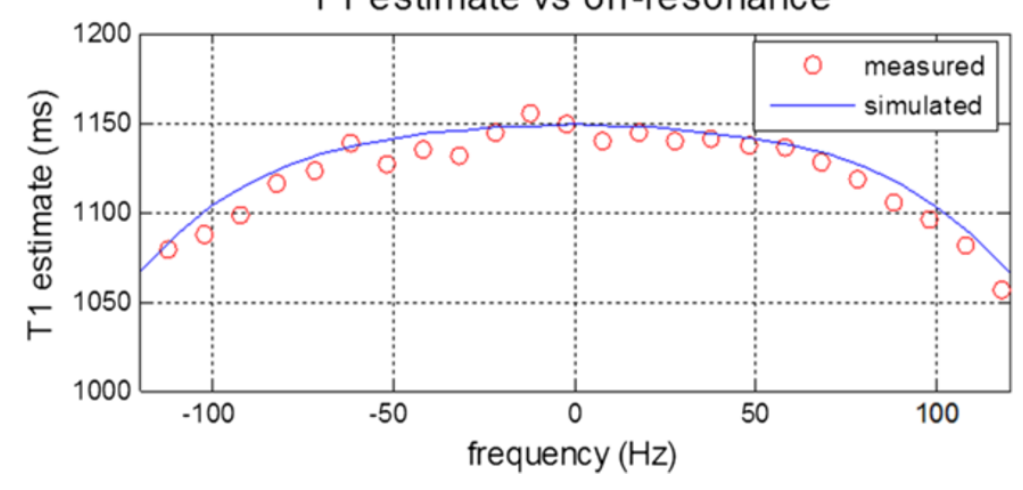

Figure 4 Off-resonance error in $\mathrm{T} 1$ comparing phantom measurements (red) with Bloch simulation (blue) for a phantom with $\mathrm{T} 1=1197 \mathrm{~ms}$ and $\mathrm{T} 2=47 \mathrm{~ms}$ using MOLLI 5(3s)3 protocol with a $35^{\circ}$ flip angle. 

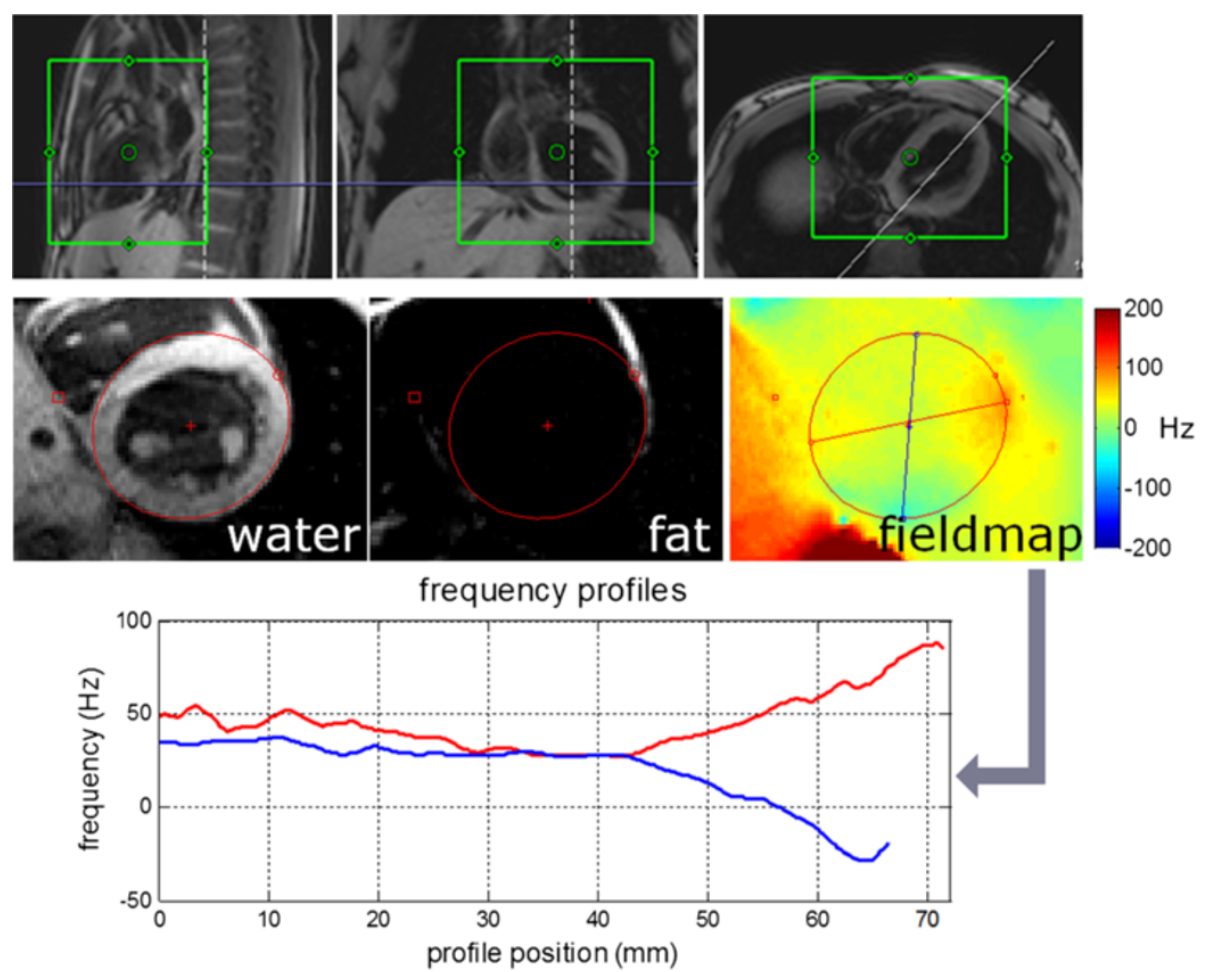

Figure 5 Local $2^{\text {nd }}$ order shim prescription and field map measurement using multi-echo GRE approach at 3T which jointly estimates water, fat, and field map. Off-resonance variation is particularly pronounced at the tissue-air interface.

(Figure 6) which is further accentuated with even small errors in center frequency.

The example in Figure 7 illustrates the trade-off between SNR and T1-measurement bias by acquiring data at different flip angles. Simulation of T1 estimates vs. measurements using $\mathrm{T} 1=1365 \mathrm{~ms}$ and $\mathrm{T} 2=45 \mathrm{~ms}$.
Using a lower flip angle (FA) trades SNR (precision) for improved accuracy and reduced off-resonance sensitivity. Reducing the flip angle from $35^{\circ}$ to $20^{\circ}$ causes a reduction in SNR from 36 to 27 in the septum and 29 to 23 in the lateral wall. The T1 estimate in the septum is $1284 \mathrm{~ms}$ at $35^{\circ}$ and 1330 at $20^{\circ}$ which agrees well with simulation

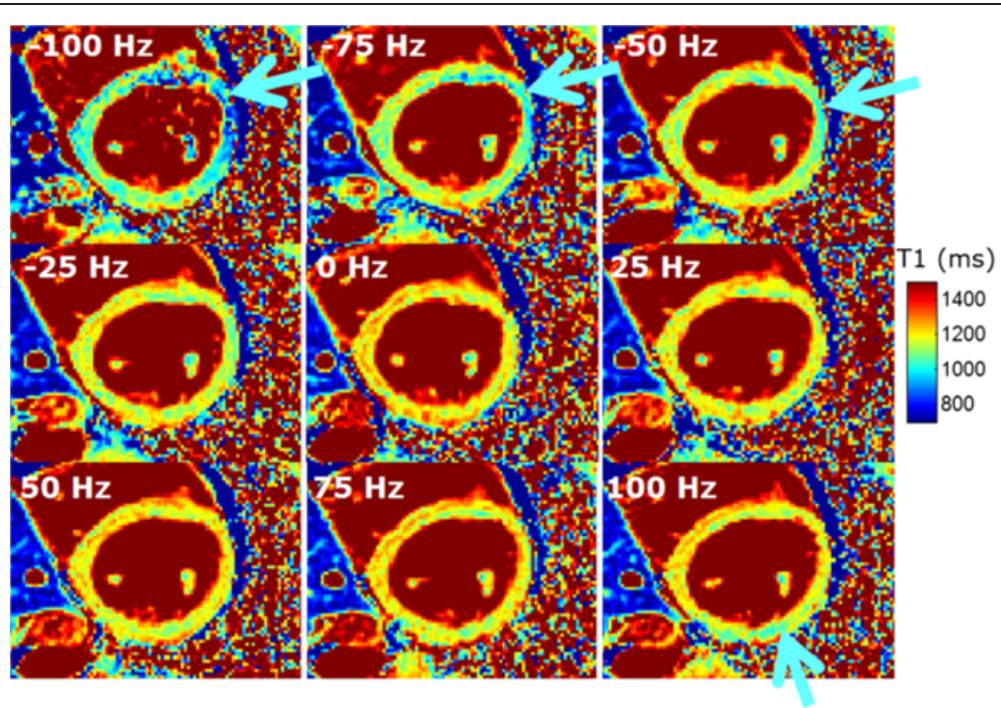

Figure 6 T1-maps acquired at different center frequencies using MOLLI at 3T. Despite the use of a $2^{\text {nd }}$ order shim in a local volume around the heart, off-resonant variation across the heart (Figure 5) leads to local variation in the apparent T1 as indicated by arrows. 


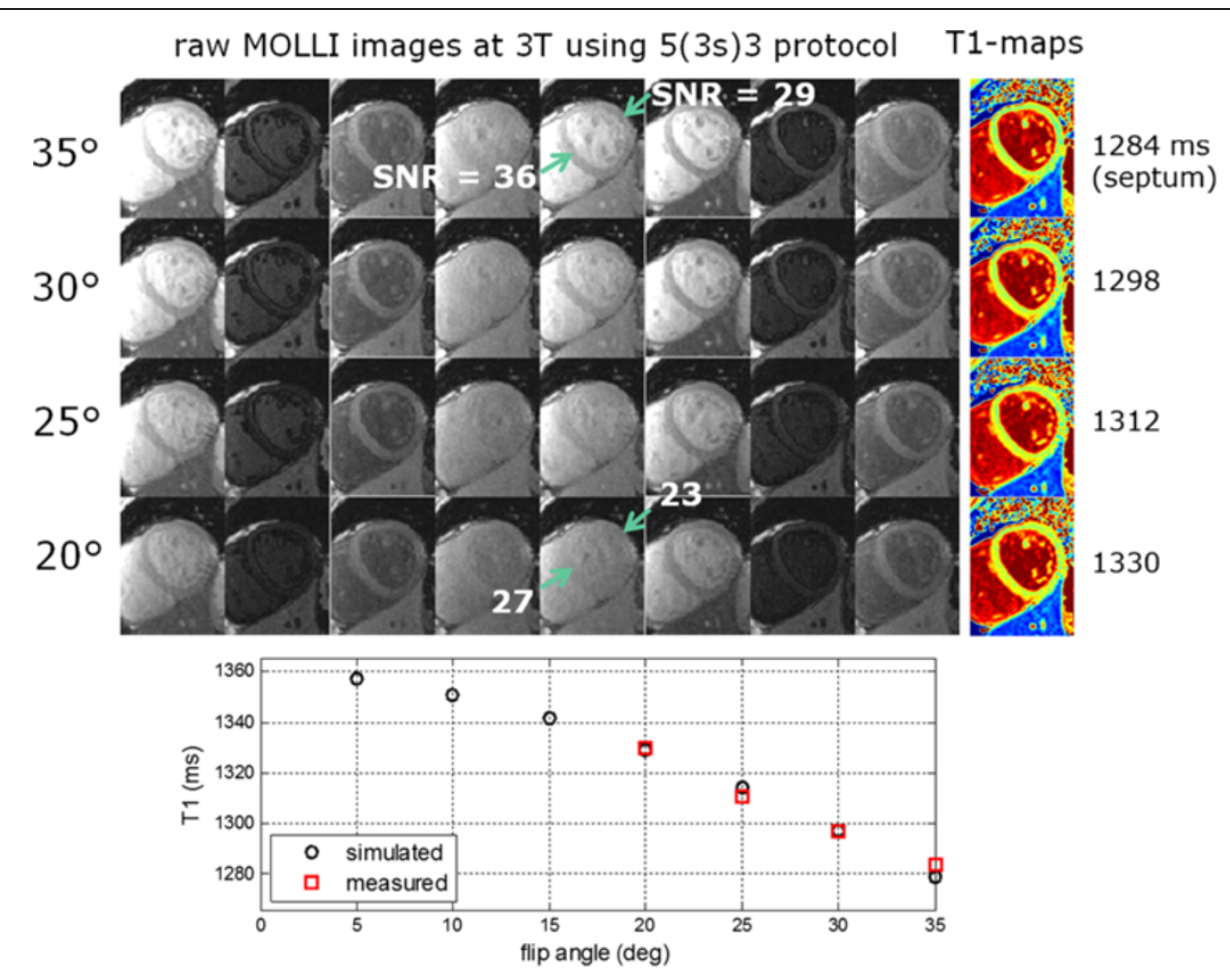

Figure 7 T1 maps and raw inversion recovery images acquired at 3T using MOLLI 5(3s)3 protocol at various flip angles illustrating tradeoff between SNR and T1-measurement bias. Simulation of T1 estimates vs measurements using $\mathrm{T} 1=1365 \mathrm{~ms}$ and $\mathrm{T} 2=45 \mathrm{~ms}$.

(Figure 7) using T1/T2 = 1365/45 ms. The T1 measurement error off-resonance is calculated by simulation (Figure 8) for this example. T1 bias error due to the effect of the readout on the inversion recovery curve is $-48 \mathrm{~ms}$ and $-81 \mathrm{~ms}$ for $\mathrm{FA}=20^{\circ}$ and $35^{\circ}$, respectively. Sensitivity to off-resonance over $\pm 100 \mathrm{~Hz}$ is $98 \mathrm{~ms}$ and $164 \mathrm{~ms}$ for $\mathrm{FA}=20^{\circ}$ and $35^{\circ}$, respectively.

\section{Discussion}

\section{T1 underestimation}

It is generally assumed that shimming and center frequency adjustment are not significant problems at $1.5 \mathrm{~T}$. Maximum off-resonance averaged $61 \pm 15.5 \mathrm{~Hz}$ across the LV $(\mathrm{n}=18)$ leading to an apparent variation in T1 of $0.5 \%$ for $\mathrm{T} 1=400 \mathrm{~ms}$, and $>1 \%$ for $\mathrm{T} 1=1000 \mathrm{~ms}$

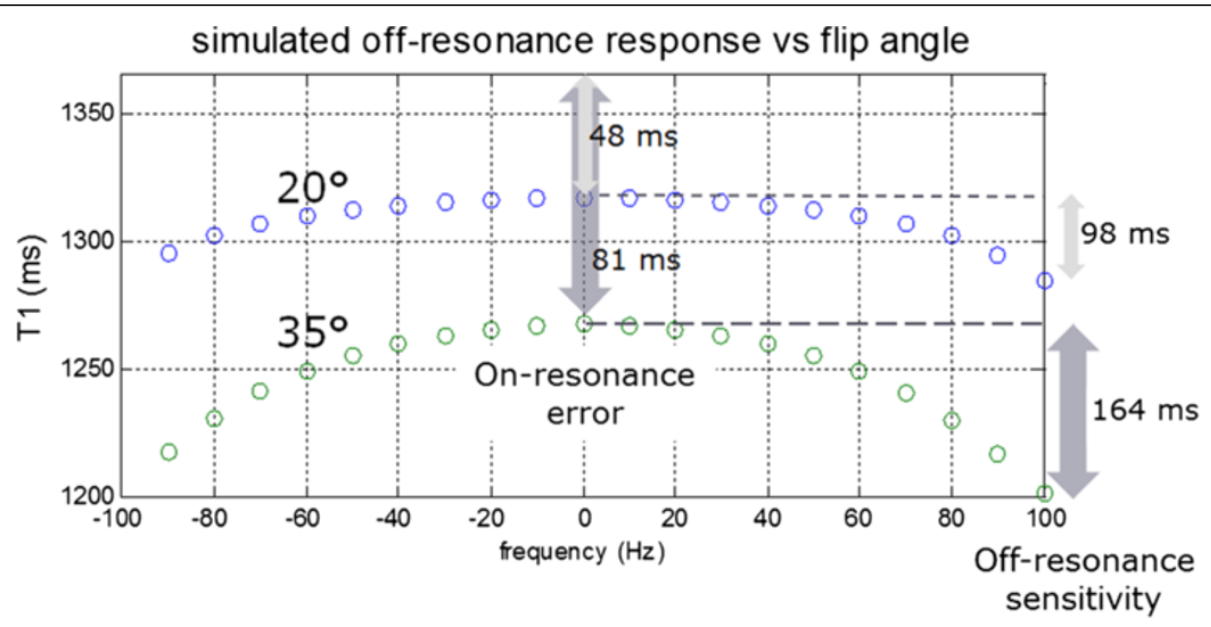

Figure 8 Using a lower flip angle (FA) trades SNR (precision) for improved accuracy and reduced off-resonance sensitivity. Simulation compares $20^{\circ}$ and $35^{\circ}$ readout flip angle for $\mathrm{T} 1=1365 \mathrm{~ms}$ and $\mathrm{T} 2=45 \mathrm{~ms}$ (representative values at $3 \mathrm{~T}$ ). T1 bias error due to the effects of readout on the inversion curve is $-48 \mathrm{~Hz}$ and $-81 \mathrm{~Hz}$ for $\mathrm{FA}=20^{\circ}$ and $35^{\circ}$, respectively. Sensitivity to off-resonance over $\pm 100 \mathrm{~Hz}$ is $98 \mathrm{~ms}$ and $164 \mathrm{~ms}$ for $\mathrm{FA}=20^{\circ}$ and $35^{\circ}$, respectively. 
(calculated using a Bloch simulation for the specific MOLLI protocol described). This represents the expected mean error in T1 $(\mathrm{n}=18)$, however, offresonance $>80 \mathrm{~Hz}$ was observed in 4 of 18 subjects, which resulted in more significant $\mathrm{T} 1$ errors $(>3 \%)$ which could be confused with regional variation in T1 due to pathology. In a large study of normal subjects [20], the T1 measurements were reported to have standard deviation $<2 \%$ at $1.5 \mathrm{~T}$. However, that study measured the mean per subject across the heart which tends to downplay regional variation due to local susceptibility. At 3.0T the off-resonance is typically greater, with mean off-resonance found to be $\sim 125 \mathrm{~Hz}$ across the LV, after local shimming. The variation in $\mathrm{T} 1$ at $3 \mathrm{~T}$ from onresonance with this protocol was calculated to be approximately $3 \%$ at $125 \mathrm{~Hz}$ for T1 $=400 \mathrm{~ms}$, and $>5 \%$ at $125 \mathrm{~Hz}$ for $\mathrm{T} 1=1000 \mathrm{~ms}$. Off-resonance $>150 \mathrm{~Hz}$ was observed (7 of 18 subjects) leading to significant regional variation.

In this study, the readout size was 256 sampled in the frequency direction in order to improve the spatial resolution to help mitigate partial volume effects at tissue interfaces, e.g, myocardium and blood. This readout length results in a TR of $2.8 \mathrm{~ms}$. A shorter TR (and readout) would somewhat decrease the sensitivity to off-resonance. The off-resonance sensitivity is also dependent on the number of RF pulses to the center of $\mathrm{k}$-space and total number of readouts, and can be reduced by decreasing the matrix size and greater partial Fourier. Modifying the protocol in this way may result in lower spatial resolution and degraded point spread function leading to T1-errors caused by greater partial volume contamination of the myocardium by the adjacent blood pool.

The use of separate reference line auto-calibration of parallel imaging eliminates 12 phase encodes per shot (6 to center of k-space) using the current widely used in-place calibration approach. For this reason, we have recently adopted a separate reference line approach which acquires the auto-calibration data at the end of the imaging acquisition such that the reference lines do not influence the initial magnetization. This has the additional benefit of reducing the overall shot time by $34 \mathrm{~ms}$ which is particularly important at higher heart rates to reduce cardiac motion blur.

Reduction in excitation flip angle can reduce the offresonance sensitivity at the cost of decreased SNR. Reduction in flip angle also reduces the absolute bias error (i.e., on-resonance error). Using a FA $=35^{\circ}$ the SNR is in the range 20-40 across the LV and the precision of T1 estimates is between 45 and $22 \mathrm{~ms}$ (SD measured on a per pixel basis), respectively, using the 5(3)3 protocol [21]. By reducing the flip angle from $35^{\circ}$ to $20^{\circ}$ the SNR is reduced by approximately $40 \%$ to the range of $15-30$ across the LV. With this SNR reduction, the precision of
T1 estimates is 60 to $30 \mathrm{~ms}$, respectively. The precision of the $\mathrm{T} 1$ estimated in a myocardial sector is improved several fold by averaging the voxels within the sector, however, per pixel precision is still important for detecting subtle regional variation. This trade-off might be worthwhile particularly at $3 \mathrm{~T}$ where there is greater SNR to begin with and the magnitude of the off-resonance problem is expected to be greater. At some point, a FLASH based readout also becomes competitive but this requires further optimization, which is beyond the scope of this study.

\section{Off-resonance variation}

Off-resonance maps may be useful for improved confidence in measured T1-values, although it would be difficult to correct the T1 values. Values for typical off-resonance variation were established in a relatively small patient population where the variation is primarily caused by tissue air interfaces. In subjects with sternal wires or devices the off-resonance variation may increase. In severe cases with rapid spatial variation of off-resonance, an additional issue may arise in cases where the subject has difficulty breath-holding. In this case, the possibility arises that the off-resonance variation over time will also affect the estimate of $\mathrm{T} 1$, but this case has not been studied.

Lastly, since it has been shown that the sensitivity to off-resonance is a function of flip angle, there is a complex interaction between the variation in off-resonance due to B0-inhomogeneity and the variation in actual flip angle due to B1 transmit inhomogeneity. The B0 and $\mathrm{B} 1+$ field inhomogeneities are fairly independent, however sensitivity of $\mathrm{T} 1$ estimate to variation in flip angle has not been studied.

\section{Off-resonance sensitivity of ECV}

Errors in T1 due to off-resonance may also influence the calculation of ECV maps [10] derived from T1-maps. A preliminary analysis has shown that the systematic bias errors in ECV due to off-resonance is quite low, on the order of $1 \%$ or less (in units of ECV percentage). Although the blood T1 is much longer, errors in ECV are dominated by the pre-contrast $\mathrm{T} 1$. Interestingly, the $\mathrm{T} 1$ measurement blood is not sensitive to off-resonance as a result of flow. The source of error in the stationary myocardial tissue T1 estimate using the MOLLI method is due to the approximation of the so called Look-Locker correction (B/A-1) which arises due to modification of the apparent inversion recovery curve leading to a $\mathrm{T}^{*}$ * that is shorter than T1. In the case of flowing blood where there are new spins at each beat, the inversion recovery is not influenced by the previous readout and the $\mathrm{T} 1$ * from the 3-parameter exponential fit provides and an accurate estimate of $\mathrm{T} 1$. 


\section{Conclusions}

The use of single shot SSFP imaging for T1-mapping in the heart is accompanied by an off-resonance sensitivity of the T1 estimates. Significant T1 measurement errors may arise well within the SSFP "passband" in regions that do not experience dark band artifacts. The off-resonant behavior is due to the transient approach to steady state, which depends on the initial magnetization determined by the inversion recovery. The apparent $\mathrm{T} 1$ depends on offresonance leading to a T1-underestimation. Off resonance errors worsen with higher excitation flip angles. Regional variations due to the inability to completely shim the B0-field variation around the heart appear as regional variation in $\mathrm{T} 1$, which is artifactual.

\section{Abbreviations \\ CMR: Cardiovascular Magnetic Resonance; MOLLI: modified Look-Locker inversion recovery; TI: Inversion time; ROI: Region-of-interest.}

\section{Competing interests}

Dr. Arai is a principal investigator on a US government Cooperative Research and Development Agreement (CRADA) with Siemens Medical Solutions (HL-CR-05-004).

\section{Authors' contributions}

PK conceived of the study, wrote the simulation, performed measurements and analysis, and drafted the manuscript. DAH contributed to the Bloch simulations. AEA had overall responsibility for human studies, MSH contributed to the interpretation of results. All authors participated in revising the manuscript and read and approved the final manuscript.

\section{Funding}

Supported by the National Heart, Lung and Blood Institute, National Institutes of Health by the Division of Intramural Research, NHLBI, NIH, DHHS (HL004607-14CPB).

\section{Author details}

${ }^{1}$ National Heart, Lung, and Blood Institute, National Institutes of Health, DHHS, 10 Center Drive MSC-1061, Bethesda, MD 20892, USA. ${ }^{2}$ Biomedical Engineering, Johns Hopkins University School of Medicine, Baltimore, MD, USA.

Received: 4 June 2013 Accepted: 7 July 2013

Published: 22 July 2013

\section{References}

1. Bieri O, Scheffler K. Fundamentals of balanced steady state free precession MRI. J Magn Reson Imaging. 2013. doi:10.1002/jmri.24163 [Epub ahead of print].

2. Wieben O, Francois C, Reeder SB. Cardiac MRI of ischemic heart disease at 3T: potential and challenges. Eur J Radiology. 1008; 65(1):15-28.

3. Schar M, Kozerke S, Fischer SE, Boesiger P. Cardiac SSFP imaging at 3 Tesla. Magn Reson Med. 2004; 51:799-806.

4. Messroghli DR, Radjenovic A, Kozerke S, Higgins DM, Sivananthan MU, Ridgway JP. Modified look-locker inversion recovery (MOLLI) for highresolution T1 mapping of the heart. Magn Reson Med. 2004; 52:141-46.

5. Messroghli DR, Greiser A, Frohlich M, Dietz R, Schulz-Menger J. Optimization and validation of a fully-integrated pulse sequence for modified look-locker inversion-recovery (MOLLI) T1 mapping of the heart. J Magn Reson Imaging. 2007; 26:1081-6.

6. Flett AS, Hayward MP, Ashworth MT, et al. Equilibrium contrast cardiovascular magnetic resonance for the measurement of diffuse myocardial fibrosis: preliminary validation in humans. Circulation. 2010; 122(2):138-44

7. Sado DM, Flett AS, Moon JC. Novel imaging techniques for diffuse myocardial fibrosis. Future Cardiol. 2011; 7(5):643-50.
8. Schelbert E, Testa SM, Meier CG, et al. Myocardial extracellular volume fraction measurement by gadolinium cardiovascular magnetic resonance in humans: slow infusion versus bolus. J Cardiovasc Magn Reson. 2011; 13:16.

9. Ugander M, Oki AJ, Hsu L-Y, et al. Extracellular volume imaging by MRI provides insight into overt and subclinical myocardial pathology. Eur Heart J. 2012; 33(10):1268-78.

10. Kellman $P$, Wilson JR, Xue $H$, Ugander M, Arai AE. Extracellular volume fraction mapping in the myocardium, Part 1: evaluation of an automated method. J Cardiovasc Magn Reson. 2012; 14:63.

11. Kellman P, Wilson JR, Xue H, Bandettini WP, Shanbhag SM, Druey KM, Ugander M, Arai AE. Extracellular volume fraction mapping in the myocardium, Part 2: initial Clinical Experience. J Cardiovasc Magn Reson. 2012; 14:64.

12. Karamitsos TD, Piechnik SK, Banypersad SM, et al. Noncontrast T1 mapping for the diagnosis of cardiac amyloidosis. JACC CardiovasC Imaging. 2013 (13):00138-1. doi:10.1016/j.jcmg.2012.11.013. pii: S1936-878X, [Epub ahead of print].

13. Bull S, White SK, Piechnik SK, et al. Human non-contrast T1 values and correlation with histology in diffuse fibrosis. Heart. 2013 [Epub ahead of print].

14. Sado DM, White SK, Piechnik SK, et al. The identification and assessment of Anderson fabry disease by cardiovascular magnetic resonance noncontrast myocardial T1 mapping. Circ Cardiovasc Imaging. 2013 [Epub ahead of print].

15. Deichmann R, Haase A. Quantification of TI Values by SNAPSHOT-FLASH NMR Imaging. J Magn Reson. 1992; 612:608-12.

16. Gai ND, Stehning C, Nacif M, Bluemke DA. Modified Look-Locker T(1) evaluation using Bloch simulations: Human and phantom validation. Magn Res Med. 2012. doi:10.1002/mrm.24251 [Epub ahead of print].

17. Kellman P, Herzka DA, Hansen MS. Adiabatic inversion pulses for myocardial T1-mapping. Magn Res Med. 2013. doi:10.1002/mrm.24793 [Epub ahead of print].

18. Deshpande VS, Chung YC, Zhang Q, Shea SM, Li D. Reduction of transient signal oscillations in True-FISP using a linear flip angle series magnetization preparation. Magn Reson Med. 2003; 49:151-57.

19. Hernando D, Kellman P, Haldar JP, Liang Z-P. Robust water/fat separation in the presence of large field inhomogeneities using a graph cut algorithm. Magn Reson Med. 2010; 63(1):79-90.

20. Piechnik SK, Ferreira VM, Lewandowski AJ, Ntusi NA, Banerjee R, Holloway C, Hofman MB, Sado DM, Maestrini V, White SK, Lazdam M, Karamitsos T, Moon JC, Neubauer S, Leeson P, Robson MD. Normal variation of magnetic resonance $T 1$ relaxation times in the human population at 1.5 T using ShMOLLI. J Cardiovasc Magn Reson. 2013; 15:13.

21. Kellman $P$, Arai $A E, X$ Ue $H . T 1$ and extracellular volume mapping in the heart: estimation of error maps and the influence of noise on precision. J Cardiovasc Magn Reson. 2013; 15(1):56.

doi:10.1186/1532-429X-15-63

Cite this article as: Kellman et al:: Influence of Off-resonance in myocardial T1-mapping using SSFP based MOLLI method. Journal of Cardiovascular Magnetic Resonance 2013 15:63.

\section{Submit your next manuscript to BioMed Central and take full advantage of:}

- Convenient online submission

- Thorough peer review

- No space constraints or color figure charges

- Immediate publication on acceptance

- Inclusion in PubMed, CAS, Scopus and Google Scholar

- Research which is freely available for redistribution 